\title{
Retransplantation after post transplant lymphoproliferative disorder: overcoming the obstacles!
}

\author{
Pallavi Prasad ${ }^{1}$ - Dinesh Khullar ${ }^{1}$ (D) Nimish Gupta ${ }^{1} \cdot$ Rahul Grover $^{1} \cdot$ Gagandeep Chhabra $^{1} \cdot$ Kunal Raj Gandhi $^{1}$. \\ Sagar Gupta ${ }^{1,3} \cdot$ Sahil Bagai $^{1}$
}

Received: 27 August 2019 / Accepted: 24 January 2020 / Published online: 3 February 2020

(c) Japanese Society of Nephrology 2020

\begin{abstract}
Post transplant lymphoproliferative disorder (PTLD) is a rare complication after kidney transplantation. Graft dysfunction is often encountered during the course of the treatment of PTLD, at times leading to need for retransplantation. We describe here the case of a young boy who underwent retransplantation after treatment of early Epstein Barr virus (EBV) related post transplant lymphoproliferative disorder. Our case highlights the various factors needing deliberation before retransplantation including time from remission of PTLD, EBV serostatus and choice of induction and maintenance immunosuppression agents.
\end{abstract}

Keywords Post transplant lymphoproliferative disorder $\cdot$ EBV $\cdot$ Retransplantation

\section{Introduction}

Post transplant lymphoproliferative disorder (PTLD), although rare, is one of the most common post transplant malignancies in kidney transplant recipients [1,2]. Net state of immunosuppression and viral infections (especially EBV infection), are the two most important risk factors leading to development of PTLD [1]. Retransplantation after PTLD is a challenge due to the risks of immunosuppression with induction and maintenance agents on one hand and managing a potentially sensitized kidney transplant recipient on the other hand. We describe here the case of a young boy who underwent successful kidney retransplantation following treatment for post transplant lymphoproliferative disorder.

Dinesh Khullar

drdineshkhullar@gmail.com

1 Department of Nephrology, Max Super Speciality Hospital, 2, Press Enclave Road, Saket, New Delhi 110017, India

2 Present Address: Department of Nephrology, Sri Ramachandra Medical College, Porur, Chennai, India

3 Present Address: Department of Nephrology, Metro Heart Institute with Multispeciality, Faridabad, Haryana, India

\section{Case report}

A 12-year-old boy, a case of end stage renal disease secondary to obstructive uropathy, underwent live related kidney transplantation with his mother as a donor. Induction agent used was basiliximab and maintenance agents were tacrolimus, mycophenolate sodium and prednisolone. He had an uneventful intraoperative course but developed an active $\mathrm{T}$ cell mediated rejection (TCMR) on post operative day three. Despite three doses of methylprednisolone, graft function continued to worsen and he was given rabbit-antithymocyte globulin (ATG), a total dose of $9 \mathrm{mg} / \mathrm{kg}$, for steroid resistant TCMR. Graft function improved rapidly and he achieved a nadir serum creatinine of $0.9 \mathrm{mg} / \mathrm{dl}$. He maintained stable graft function subsequently. One year later, he presented with symptoms of throat irritation and right sided ear discharge. After inadequate response with antibiotics and antihistaminics, he underwent a nasal endoscopy which revealed a friable nasopharyngeal mass. Biopsy of the mass was suggestive of a diffuse large B cell lymphoma. The large cells were $\mathrm{CD} 20$ positive, CD3 negative and EBV latent membrane protein 1 positive. PET scan did not show involvement of the allograft but there was involvement of inguinal lymph nodes on the right side. CSF and bone marrow biopsy were normal. Based on the above findings, a diagnosis of Stage III EBV related early monomorphic posttransplant lymphoproliferative disorder (PTLD) was made. His immunosuppression was changed to reduced dose tacrolimus, everolimus 
and steroid. Cyclical chemotherapy (rituximab, cyclophosphamide, doxorubicin, vincristine and prednisolone) and radiotherapy were started. He achieved complete disease remission within 3 months of starting chemotherapy.

Two months after completing chemotherapy, he developed a rise in serum creatinine to $2 \mathrm{mg} / \mathrm{dl}$. Allograft biopsy was suggestive of an acute thrombotic microangiopathy (TMA) with five glomeruli showing fibrin thrombi in capillary lumen and one arteriole showing endothelial swelling and fibrinoid necrosis. Tubulopathic changes like epithelial simplification and vacuolisation were also present. Nodular perioidic acid Schiff (PAS) positive arteriolar hyalinosis was present and $\mathrm{C} 4 \mathrm{~d}$ stain by immunoflourescence was negative in peritubular capillaries. Investigations for determining the cause of thrombotic microangiopathy were done but were inconclusive. Donor-specific antibodies were undetectable and blood did not show any evidence of viremia (polymerase chain reaction tests in blood for CMV and EBV were negative). Suspecting drug induced TMA, immunosuppression was changed from tacrolimus to cyclosporine and from everolimus to mycophenolate sodium. However, graft function continued to worsen. As a last resort, patient was started on plasmapheresis with low dose intravenous immunoglobulin in suspicion of antibody mediated rejection related TMA. There was an inadequate response to plasmapheresis and over the next 2 years the patient had a gradual rise in serum creatinine and became dialysis dependent.

The patient was planned for a second kidney transplantation two years after having achieved complete remission of PTLD with his father being the prospective donor. Pretransplant PET scan was negative for fluorodeoxyglucose (FDG) avid lesions. However, he continued to show PCR positivity for EBV (100 copies/ml) in serum and EBV serology showed IgG negative status. Given the high risk of recurrence of PTLD with positive EBV viremia [2], we decided against retransplantation at this stage. Monthly monitoring of EBV PCR and EBV serology was done. Six months later, we were able to document a complete clearance of EBV viremia and a positive immune response against $E B V$ in the form of positive EBV IgG antibodies (against EBV nuclear antigen). Donor specific antibodies were undetectable and both flow and complement dependent cytotoxicity cross match tests were negative (Table 1). In retransplantation we decided to use basiliximab as the induction agent for the second time to avoid the risks involved with rechallenging with thymoglobullin in view of a past history of PTLD. He was also given rituximab $(100 \mathrm{mg})$ before transplantation to prevent EBV reactivation. The patient underwent successful kidney retransplantation surgery 33 months after achieving complete remission of PTLD. He is currently maintained on tacrolimus, mycophenolate sodium and prednisolone and valacyclovir prophylaxis for the prevention of EBV reactivation. EBV PCR is being monitored 6 monthly for early
Table 1 Investigations done prior to retransplantation

Investigations done prior to retransplantation
Hemoglobin $9.8 \mathrm{~g} / \mathrm{dl}$

WBC count $7800 / \mathrm{mm}^{3}$

Platelet count $1.8 \mathrm{lakh} / \mathrm{mm}^{3}$

Serum LDH 120 IU/L

PET CT negative for FDG avid

lesions

EBV IgG donor + recipient +

CMV PCR negative
EBV PCR negative
CMV IgG donor + recipient + HBsAg negative Anti HCV antibody negative HCV RNA negative HIV antibody negative DSA No DSA detected CDC cross match negative Flow cross match negative detection of reactivation of EBV. Two years post retransplantation, he is maintaining a stable graft function, is free of any opportunistic infections and is in complete remission of lymphoma.

\section{Discussion}

Post transplant lymphoproliferative disorder (PTLD) carries adverse implications for both patient and graft survival post kidney transplantation [1].Risk factors for PTLD include extremes of age (both paediatric and older recipients $>60$ years old), use of $\mathrm{T}$ cell depleting antibodies (for induction or rejection), tacrolimus use, net maintenance immunosuppression and EBV seronegativity or EBV seromismatch (transplantation of organs from an $\mathrm{EBV}+$ donor into an EBV-recipient) [1, 2]. The corner stone of therapy is reduction in immunosuppression albeit associated with a higher risk of rejection, as may have happened in our patient. Other strategies include use of antiviral agents, EBV-specific cytotoxic T lymphocytes, chemotherapy including rituximab, surgical resection and radiation [1].

Prevalence of PTLD in kidney transplant recipients in Asia varies between 0.75 and 1.9\% [3-5]. Recent studies show that late PTLD is more common than early PTLD, diffuse large B cell lymphoma is the most common pathological variant [5]. Although most cases of early PTLD are EBV related, up to $40 \%$ of late PTLD may also show EBV positivity [5]. Rates of graft loss up to $50 \%$ [3, 4] have been reported in PTLD patients and may be due to acute rejection, chronic rejection or due to opportunistic infections after chemotherapy [1]. Hence, the importance of retransplantation in these patients needs to be emphasised. To the best of our knowledge, this is the first reported case of kidney retransplantation post remission of PTLD from Asia.

Two case series report the results of kidney retransplantation following graft loss in PTLD patients: one from United network for organ sharing (UNOS) in USA and one from a French cohort of PTLD patients [1, 2]. In the largest series from France, 55 kidney retransplantations were done and in the OPTN/UNOS Database Study by Johnson et al., 22 
kidney retransplantations were performed after graft loss following treatment for PTLD.

The ideal time gap between PTLD remission and listing for retransplantation is not clear. The British Transplantation Society guidelines recommend a period of at least 1 year from the control of PTLD to retransplantation to minimize the risk of PTLD recurrence [6].In the French registry, the median time from PTLD to listing for retransplantation was 65 months and from PTLD to retransplantation was 90 months [2]. Recurrence of PTLD occurred in one patient in the French registry who had undergone second kidney transplantation surgery 28 months after PTLD [2]. Our patient underwent a second kidney transplantataion surgery at 36 months post PTLD diagnosis and 33 months after having achieved complete remission.

EBV status at the time of retransplantation is an important predictor of recurrence after retransplantation. It has been suggested that patients should present an EBV seroconversion (an EBV IgG seropositive status) before retransplantation and it is advisable that EBV viral load be undetectable at the time of retransplantation [7]. Antiviral prophylaxis may decrease the risk of EBV reactivation by $83 \%$ after transplantation, the effect being most prominent in the first year post transplantation [8]. Patients with low level EBV viremia at the time of retransplantation may benefit from antivirals with intermittent measurements of EBV viral load and subsequent immunosuppression adjustments as per the viral load [7]. Rituximab has also been used to prevent EBV viremia in EBV seronegative kidney transplant recipients [9].

Most patients undergoing retransplantation after PTLD receive an induction agent [2]. IL-2R antagonists are the favoured induction agents (30-40\%) in most cases [1,2]. Although antilymphocyte globulins have been used in cases of retransplantation after PTLD, it is noteworthy that in the data analysis by Johnson et al., none of the patients who received a $T$ cell depleting agent in the first transplantation were rechallenged by a $\mathrm{T}$ cell depleting agent during retransplantation [1]. Rituximab has been used in retransplantations after PTLD both as a desensitisation protocol in high risk sensitised cases and as a prophylaxis for proliferation of EBV in cases with high viral counts [2,9]. Our patient had received basiliximab as induction agent in the first transplantation but thymoglobulin had to be given for steroid resistant TCMR. In retransplantation, our patient received basiliximab and rituximab. Rituximab in our case was given to help prevent the reactivation of EBV.

Maintenance immunosuppression in retransplantation post PTLD is generally the standard combination of calcineurin inhibitor, mycophenolate sodium and prednisolone [2]. The overall state of immunosuppression determines the risk of PTLD and hence it may be advisable to keep low target levels of maintenence agents in these patients [2].
Mycophenolate sodium is the only agent seen to have a significant protective effect against PTLD and its use is common in most series [2]. mTOR inhibitors deserve a special mention in cases of PTLD. Although these agents have antiproliferative effects and have been used in combination with chemotherapy for treatment of PTLD, there is not enough evidence to recommend their use to prevent PTLD [2, 9].

\section{Conclusion}

Graft loss after PTLD treatment often calls for retransplantation. Time from PTLD remission, EBV serostatus and EBV viremia are important considerations before retransplantation. Both induction and maintenance immunosuppression should be chosen wisely so as to balance the risk of reactivation of EBV against the risk of rejection. Rituximab and antivirals are part of the armamentarium against EBV related PTLD and may be used in selected cases. Although recurrence of PTLD is rare, close monitoring of all such patients is advisable.

\section{Compliance with ethical standards}

Conflict of interest All the authors have declared no competing interest.

Informed consent Informed consent was obtained from all individual participants included in the study.

Ethical approval This article does not contain any studies with human participants performed by any of the authors.

\section{References}

1. Johnson SR, Cherikh WS, Kauffman HM, et al. Retransplantation after post-transplant lymphoproliferative disorders: an OPTN/ UNOS database analysis. Am J Transplant. 2006;6:2743-9.

2. Caillard S, Cellot E, Dantal J, et al. A French cohort study of kidney retransplantation after post-transplant lymphoproliferative disorders. Clin J Am Soc Nephrol. 2017;12:1663-700.

3. Abe T, Ichimaru N, Kokado Y, et al. Post-transplant lymphoproliferative disorder following renal transplantation: a single-center experience over 40 years. Int J Urol. 2010;17(1):48-544.

4. Chan TS, Hwang YY, Gill H, et al. Post-transplant lymphoproliferative diseases in Asian solid organ transplant recipients: late onset and favorable response to treatment. Clin Transplant. 2012;26(5):679-83.

5. Cheung CY, Ma MKM, Chau KF, et al. Posttransplant lymphoproliferative disorders in kidney transplant recipients: a retrospective cohort analysis over two decades in Hong Kong. Oncotarget. 2017;8(57):96903-12.

6. Parker A, Bowles K, Bradley A, et al. Haematooncology task force of the British Committee for Standards in Haematology and British Transplantation Society: Management of 
post-transplant lymphoproliferative disorder in adult solid organ transplant recipients - BCSH and BTS guidelines. Br J Haematol. 2010;149:693-705.

7. Hanto DW. Retransplantation after post-transplant lymphoproliferative diseases (PTLD): when is it safe? Am J Transplant. 2004;4:1733-4.

8. Funch DP, Walker AM, Schneider G, et al. Ganciclovir and acyclovir reduce the risk of post-transplant lymphoproliferative disorder in renal transplant recipients. Am J Transplant. 2005;5:2894-900.
9. Reddy N, Rezvani K, Barrett J, et al. Strategies to prevent EBV reactivation and posttransplant lymphoproliferative disorders (PTLD) after allogeneic stem cell transplantation in high-risk patients. Biol Blood Marrow Transplant. 2011;17(5):591-7.

Publisher's Note Springer Nature remains neutral with regard to jurisdictional claims in published maps and institutional affiliations. 